\title{
Friction parameters identification and compensation of LuGre model base on genetic algorithms
}

\author{
Yuqin Wen ${ }^{\mathrm{a}}$, Ming Chu ${ }^{\mathrm{b}}$ and Hanxu Sun ${ }^{\mathrm{c}}$ \\ School of Automation, \\ Beijing University of Posts and telecommunications \\ No.10 Xitucheng Road, Haidian District, Beijing, China \\ awyq_bupt@163.com
}

\begin{abstract}
According to the interference of the servo system, a method is proposed that identify the parameters of LuGre friction model and compensate the friction torque base on genetic algorithms. First, establish the LuGre friction model, on the basis of the model, identify the static parameters and the dynamic parameters in turn. Second, introduce the friction compensation to the feedback control of the servo system so that to eliminate the interference and improve the system tracking accuracy and robustness.
\end{abstract}

Keywords: LuGre friction model; genetic algorithms; servo system; friction compensation.

\section{Introduction}

Friction exists in almost all of the servo systems. In high precision servo system, friction usually has a strong nonlinear character and has impact on the position control accuracy and performance implications. There are lots of friction models including static models and dynamic models. Static models like Bingham model, Stribeck model, Kamopp model and dynamic model like Dahl model, Bliman-Sorine model, LuGre model, and so on. While Genetic Algorithms is a searching mechanism that mimics the natural biological evolution, searching the optimal solution in the global scope, can solve LuGre friction model parameters identification problem well. LuGre model can well explain crawling, pre-sliding displacement, friction memory, rising static friction. Therefore, we adopt this model to analyze. In this paper, two aspects are discussed aim at the interference problems originated from friction: parameters identification and control of friction compensation[1]. With the identification result, we can compensate the friction, now that to improve the stability and control accuracy of the servo system. 


\section{Friction model}

The primary factor in measuring a friction model is whether it can describe the friction dynamic characters neighboring zero speed or not. Stribeck model handle the strong nonlinear problems well, hence, it can be considered an ideal model. As a result, LuGre model[2] applies Stribeck while solve the problems the nonlinear factors affect the friction near zero speed.

Stribeck model can be described as:

Static friction is,

$$
F_{f}(t)=\left\{\begin{array}{c}
F_{m}, F(t)>F_{m} \\
F(t),-F_{m}<F<F_{m} . \\
-F_{m}, F(t)<-F_{m}
\end{array}\right.
$$

Dynamic friction is,

$$
\begin{aligned}
& F_{f}(t)=\left[F_{c}+\left(F_{m}-F_{c}\right) e^{-\alpha_{1}|\dot{\theta}(t)|}\right] \cdot \operatorname{sgn} \dot{\theta}(t)+k_{v} \dot{\theta} . \\
& F(t)=J \dot{\theta}(t) .
\end{aligned}
$$

Where $F(t)$ is driving force; $F_{m}$ is max static friction; $F_{c}$ is coulomb friction; $k_{v}$ is viscous friction torque coeddicient; $\dot{\theta}(t)$ is angular velocity of rotation; $\alpha$ and $\alpha_{1}$ is small positive number.

It's schematic diagram as follows,

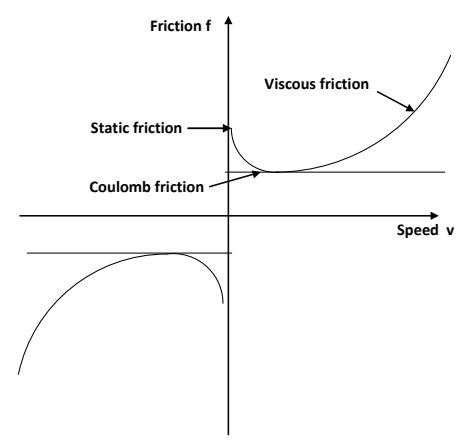

Fig. 1 Stribeck friction model diagram

The equation of the friction in servo system can be described as,

$$
m \frac{d^{2} x}{d t^{2}}=u-F \text {. }
$$

(4) 
Among this, $\mathrm{m}$ is rotational inertia, $\mathrm{x}$ is the displacement, $\mathrm{u}$ presents the control torque and $\mathrm{F}$ is a friction torque.

LuGre model is built based on the average transformation of the bristle, presented by $\mathrm{z}$, friction is $\mathrm{F}$.

$$
\begin{aligned}
& \frac{d z}{d t}=\dot{x}-\frac{\sigma_{0}|\dot{x}|}{g(\dot{x})} z . \\
& g(\dot{x})=F_{c}+\left(F_{s}-F_{c}\right) e^{-\left(\dot{x} / v_{s}\right)^{2}} . \\
& F=\sigma_{0} z+\sigma_{1} \frac{d z}{d t}+\sigma_{2} \dot{x} .
\end{aligned}
$$

Where $\sigma_{0}$ and $\sigma_{1}$ are two dynamic parameters; $F_{c}, F_{s}, v_{s}, \sigma_{2}$ are four static parameters.

When the system is in stable motion, $\frac{d z}{d t}=0$. At this time, $\mathrm{F}$ is,

$$
F=\left[F_{c}+\left(F_{s}-F_{c}\right)^{-\left(\dot{x} / v_{s}\right)^{2}}\right] \cdot \operatorname{sgn}(\dot{x})+\sigma_{2} \dot{x} .
$$

From the equation, we can see that LuGre friction model uses Stribeck model in solving the problem strong nonlinear near zero speed. Meanwhile, it describes other static and dynamic characteristics accurately.

\section{Parameters identification}

The identification of LuGre friction model is divided in two steps[3], static parameters and dynamic parameters. First, we identify four static parameters using the Stribeck curve. Second, dynamic parameters identification is proceeded based on the static parameters identification[4、5].

\section{Static parameters identification}

The closed-loop system moves at a grope of invariable speeds $\{\omega\}_{i=1}^{N}$, and the corresponding sequences of the control torque are $\{u\}_{i=1}^{N}$. From Eq. 4, we can see that, when $\frac{d^{2} x}{d t^{2}}=0, \mathrm{u}=\mathrm{F}$. Hence, the two sequences above determine the stable corresponding relations between the frictions torque and the velocity, which is 
known as the Stribeck curve. Supposed the parameter vector to be identified is $X_{s}=\left[x_{s}^{+}, x_{s}^{-}\right]$, then,

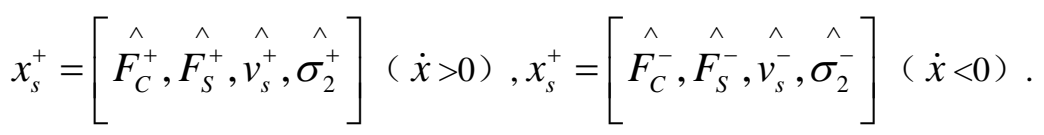

We define the error of the identification is,

$$
e\left(x_{s}, \dot{x}_{i}\right)=u_{i}-F\left(x_{s}, \dot{x}_{i}\right) \text {. }
$$

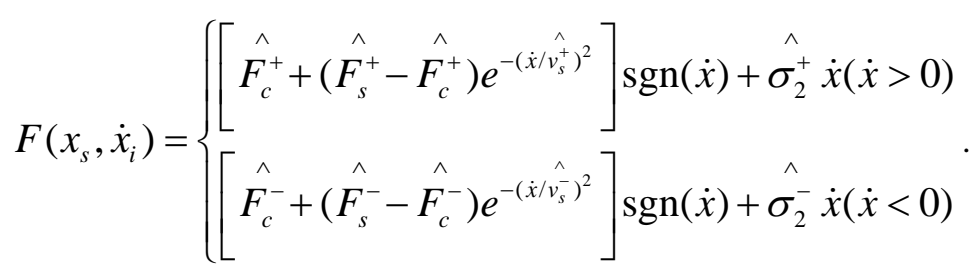

The objective function is,

$$
J=\frac{1}{2} \sum_{i}^{N} e^{2}\left(x_{s}, \dot{x}_{i}\right) \text {. }
$$

To identify the static parameters is to minimize the objective Eq. 12 .

\section{Dynamic parameters identify}

When we identify the dynamic parameters, we use the output displacement or accelerate velocity and the output control torque. Supposed the parameters is $x_{d}=\left[\hat{\sigma}_{0}, \hat{\sigma}_{1}\right]$, we define the error,

$$
e\left(x_{d}, t_{i}\right)=u\left(t_{i}\right)-u\left(x_{d}, t_{i}\right) \text {. }
$$

In the equation, $u\left(t_{i}\right)$ is the control torque of the servo system, $u\left(x_{d}, t_{i}\right)$ is the identified output control torque. Form Eq. 4, we can see,

$$
\begin{aligned}
& u\left(x_{d}, t_{i}\right)=F+m \frac{d^{2} x_{i}}{d t_{i}^{2}} . \\
& F=\hat{\sigma}_{0} z+\hat{\sigma}_{1} \dot{z}+\hat{\sigma}_{2} \dot{x} .
\end{aligned}
$$




$$
\dot{z}=\dot{x}-\frac{\hat{\sigma_{0}}|\dot{x}|}{g(\dot{x})} z .
$$

We define the objective function,

$$
J=\frac{1}{2} \sum_{i}^{N} e^{2}\left(x_{d}, t_{i}\right) \text {. }
$$

To identify the dynamic parameters is to minimize the objective Eq. 17 .

\section{The overall identity process}

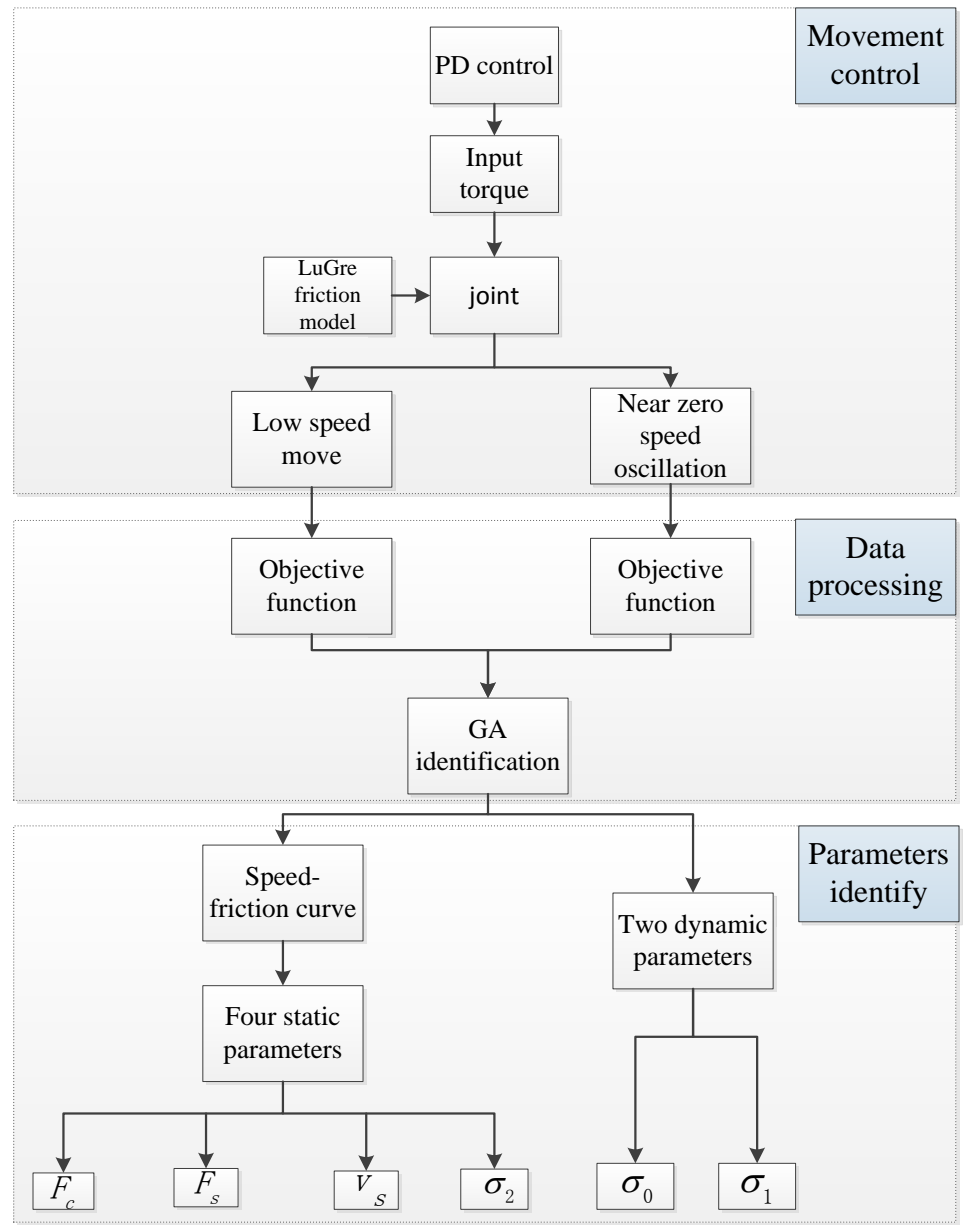

Figure. 2 GA identification process 


\section{Simulation and the results analysis}

\section{Static friction curve's test and identify}

From Eq. 4, we can see that, input a constant speed, we get u=F. Eq. 11 is adapted

friction $F_{c}^{+}=0.1503, F_{s}^{+}=0.631, \alpha^{+}=0.019, V_{s}^{+}=0.051$.

$$
F_{c}^{-}=0.201, F_{s}^{-}=0.72, \alpha^{-}=0.029, V_{s}^{-}=0.051 \quad, \quad \text { speed } \quad \text { signal }
$$

is $\left\{\dot{x}_{i}\right\}_{i=1}^{N}=[-1.0: 0.05: 1.0]$. There are 41 signals in all. Using PD control to the signals, while $k_{p}=200, k_{d}=200$. When we are tracking the speed signal, all the signals corresponds to a tracking result.

Fig. 2 is the tracking curve when the speed is one, and the result comes fairly well. Fig. 3 is the tracking error. We can tell that the error is quite little, and is approaching to zero. Fig. 4 is the curve error between practical and identified curve.

When we do the simulation by using genetic algorithms, determine population size $\mathrm{M}=100$, largest evolution algebra $\mathrm{G}=1000$. The searching area of parameters are, $F_{c} \in[0,1], F_{s} \in[0,1], V_{s} \in[0,0.1], \quad \alpha \in[0,0.1]$. Fig. 5 shows the practical curve and the identified curve. Contrast them, we can see they are closed. The result is ideal. Identification results are,

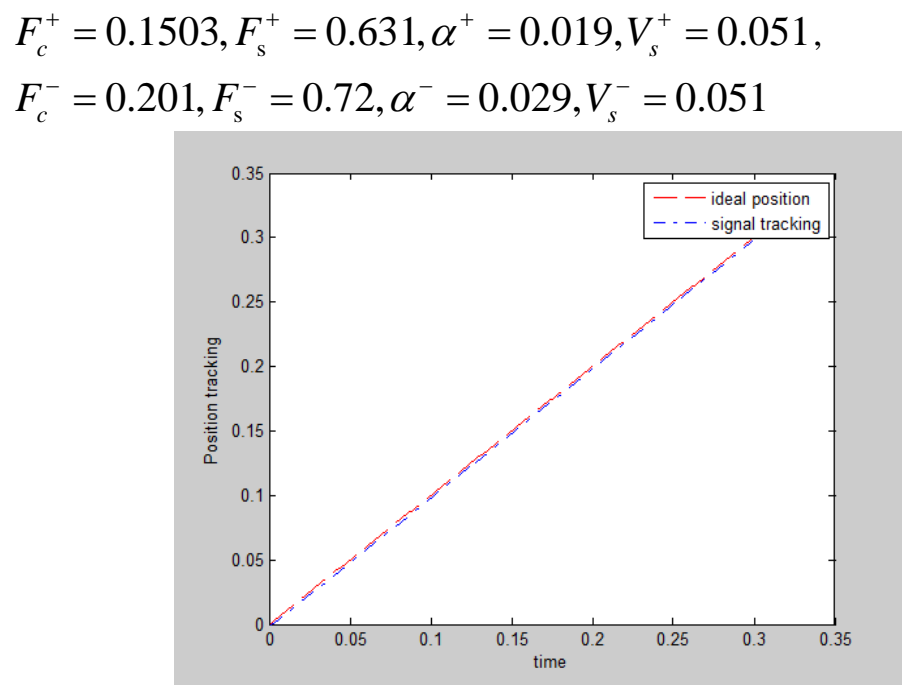

Fig. 2 speed tracking 


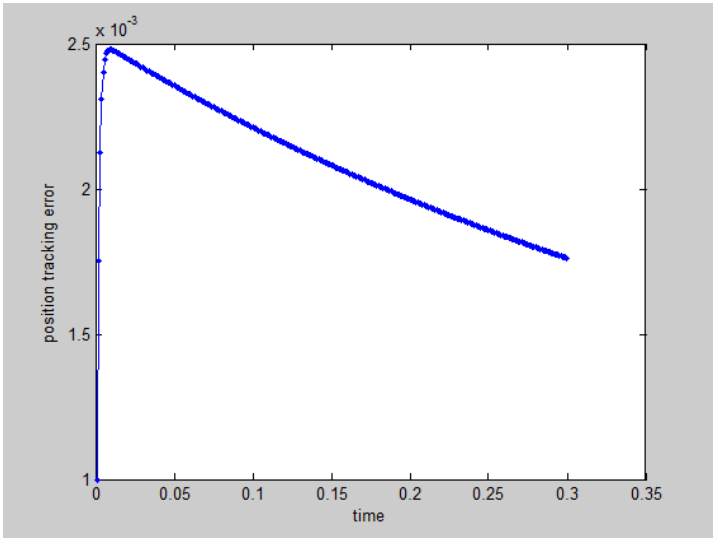

Fig. 3 speed tracking error

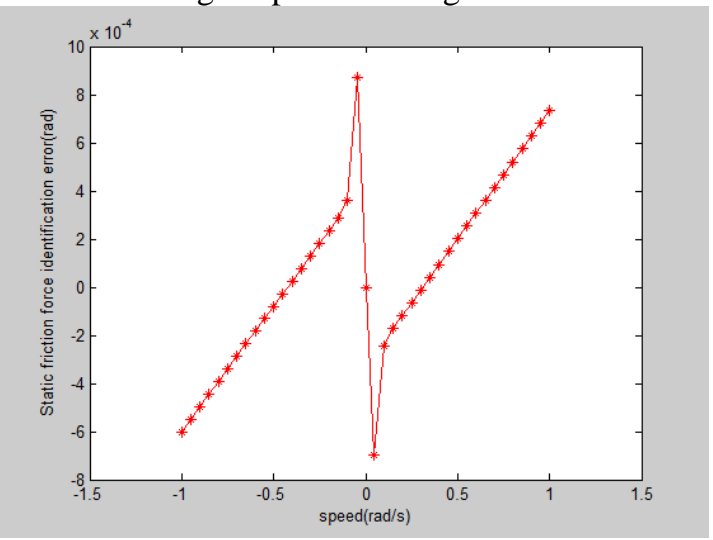

Fig. 4 friction curve error

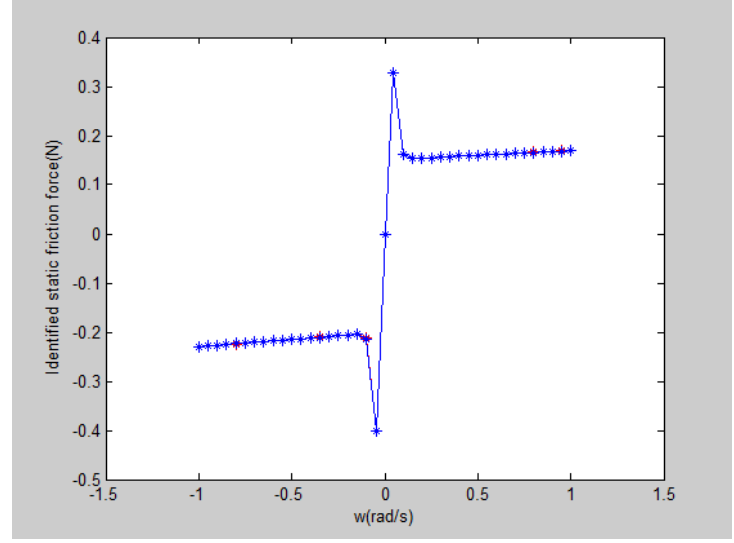

Fig. 5 practical and ideal curve 


\section{PD control of LuGre model}

According

to

Eq. 5-7,

make $J=1, \sigma_{0}=260, \sigma_{1}=2.5, F_{c}=0.28, F_{s}=0.34, V_{s}=0.01$, make the input signal sine wave $y_{d}=0.1 \sin (2 \pi t)$.

Describe the control algorithm and the system by using Simulink.

Choosing PD control, $k_{p}=20, k_{d}=5$, Fig. 4 and 5 show the tracking result of position and speed,it can obviously figure out the ceiling phenomenon. While in Fig. 6-7, we can see the serious deformation when the speed cross zero, and tracking dead zone is appeared.

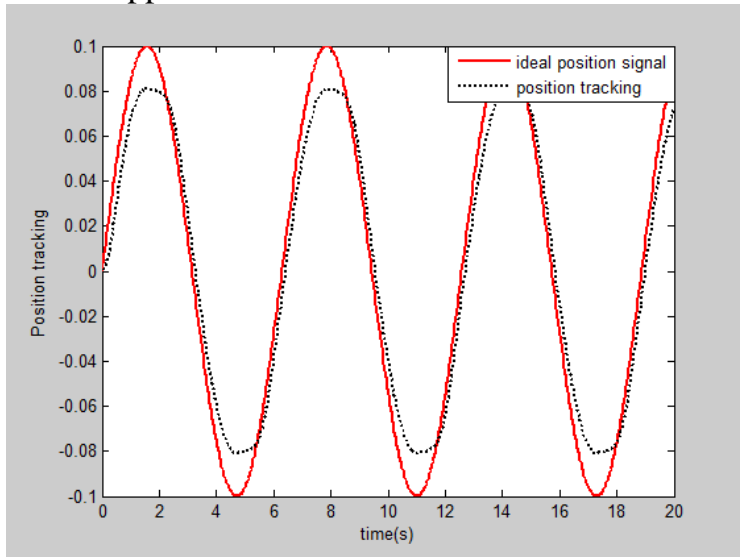

Fig. 6 position tracking

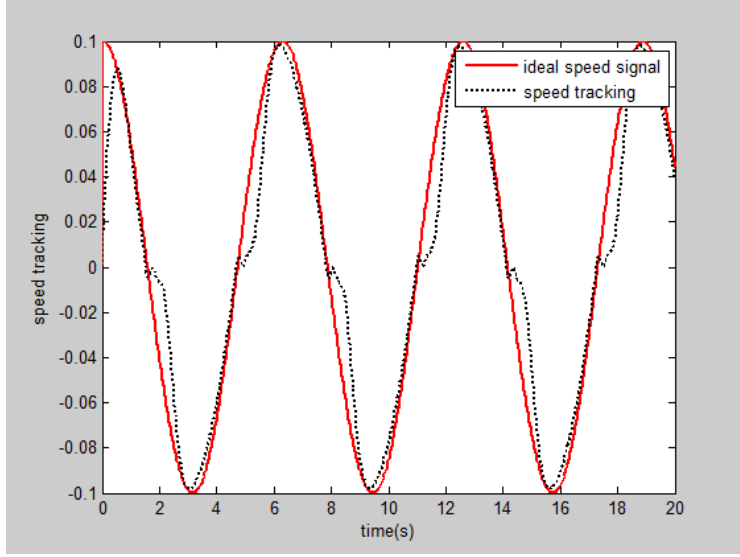

Fig. 7 speed tracking 


\section{Servo system friction compensation}

To offset the influence brought by friction, we compensate the friction according to the identification result, introducing the compensation to servo system, making it approaching the actual friction interference continuously.

Among the simulation, step signal is adopted, choosing LuGre friction model and adding in friction compensation. Using real number coding, we determine the size of sample is $30, P_{c}=0.99, P_{m}=0.10-[1: 1:$ Size $] \times 0.01 /$ Size .

During dynamic identifying, supposed the practical parameter $\sigma_{0}=0.3, \sigma_{1}=0.15$, while identify results $\sigma_{0}=0.36, \sigma_{1}=0.147$. Fig. 9 and10 are the position and speed tracking of compensation system. We can see that the speed cross zero and tracking dead zone are disappeared obviously.

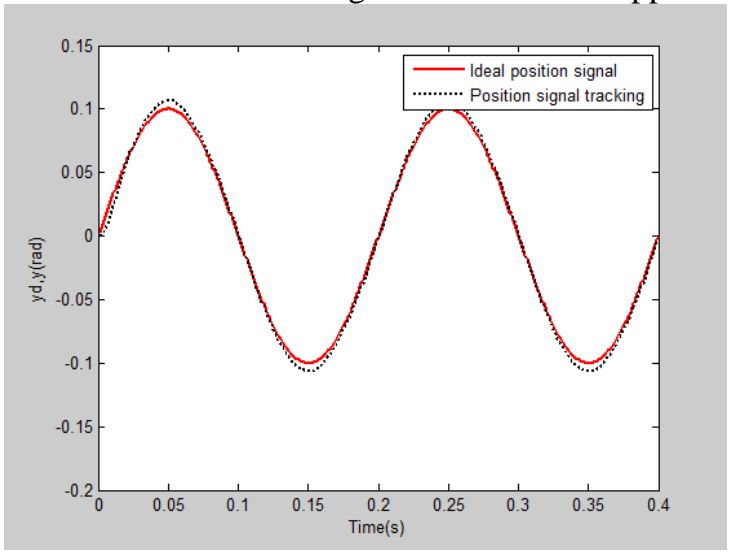

Fig. 9 position tracking with compensation

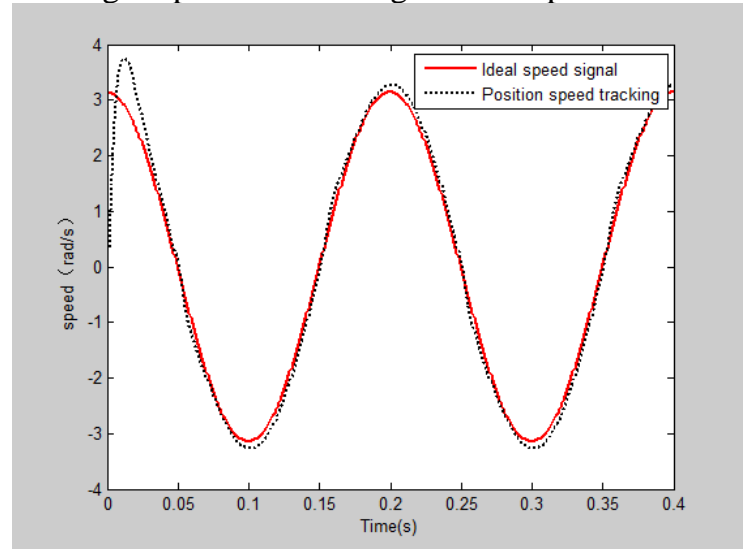

Fig. 10 speed tracking with compensation 


\section{Conclusion}

Friction is a phenomenon that cannot be avoided. It can reduce the accuracy of tracking and stability. Through the tracking result of servo system that contains friction, we can observe the effect that brought by friction. Using genetic algorithms in identifying friction parameters, can remove the effect brought by friction and making the system rapidly response thus improve the stability and tracking accuracy of the system.

\section{Acknowledgements}

This work was financially supported by Project supported by the National Natural Science Foundation of China(51305039), Scientific and technological innovation projects of Chinese PLA General Armament Department(ZYX12010001),the Fundamental Research Funds for the Central Universities(2014PTB-00-01) and National Key Basic Research Program of China (973 Program 2013CB733000).

\section{References}

[1] Wang X, Wang S, Yao B. Adaptive robust control of linear electrical loading system with dynamic friction compensation[C]//Advanced Intelligent Mechatronics (AIM), 2010 IEEE/ASME International Conference on. IEEE, 2010: 908-913.

[2] Lee $\mathrm{T} H$, Tan K K, Huang S. Adaptive friction compensation with a dynamical friction model[J]. Mechatronics, IEEE/ASME Transactions on, 2011, 16(1): 133-140.

[3] Liu D P. Research on parameter identification of friction model for servo systems based on genetic algorithms[C]//Machine Learning and Cybernetics, 2005. Proceedings of 2005 International Conference on. IEEE, 2005, 2: 1116-1120.

[4] Nakajima K, Nakajima J, Van Khoa T, et al. Identification method of nonlinear systems with friction based on Genetic Algorithm[C]//Knowledge Acquisition and Modeling (KAM), 2010 3rd International Symposium on. IEEE, 2010: 55-58.

[5] Liu D P. Parameter identification for lugre friction model using genetic algorithms[C]//Machine Learning and Cybernetics, 2006 International Conference on. IEEE, 2006: 3419-3422. 